How to reference this article

Corino Rovano, S. (2018). Le "Auree norme" del GDLI. Quando le norme redazionali diventano grammaticali. Italica Wratislaviensia, 9(1), 51-69.

DOI: http://dx.doi.org/10.15804/IW.2018.09.03

Silvia Corino Rovano

Università degli Studi di Torino

\title{
LE “AUREE NORME” DEL GDLI. QUANDO LE NORME REDAZIONALI DIVENTANO GRAMMATICALI
}

\section{THE "GOLDEN RULES" OF GDLI: WHEN STYLE SHEET BECOMES GRAMMAR}

\begin{abstract}
All dictionary editors have editorial rules. In fact, every collaborator must follow the style foreseen by the publication. To this end, every person who intends to participate in the editorial work is usually provided with a more or less consistent style sheet that contains the rules for writing the lemmas.

The editors of the GDLI were also provided with this type of material. Each collaborator, in fact, received a thin booklet entitled "Auree norme", or "Golden rules", that he had to follow. However, upon a careful examination of this precious file, the indications provided by the GDLI appear richer and more articulated than the usual set of editorial norms. In fact, they do not only contain a set of formal and typographical rules, but they have a much wider perspective: the editor is faced with a series of indications concerning spelling and grammar. The present research aims to identify the norms of grammatical interest and some elements of morphology and syntax that emerged during the discussion of the editing process of these dictionaries.
\end{abstract}

Keywords: grammar, dictionary, lexicon, rules, Italian 
La verità è che un Dizionario deve essere sempre concepito con valore di attualità. Se c'è una gerarchia da seguire o rispettare nell'ordinamento delle singole accezioni d'uno stesso vocabolo, essa non può non dipendere dalla coscienza attuale e viva che il parlante, e perciò il lessicografo, ha della parola che si definisce. [...] Noi non dobbiamo dimenticare che il nostro Dizionario non vuole essere un archivio di parole, ma una documentazione viva del linguaggio che si parla e si scrive e si esprime nella realtà contemporanea. (Criteri di lavoro, pubblicati in Bruni, 1992, pp. 124-5)

Nell'archivio del Grande Dizionario della Lingua italiana a cura di Salvatore Battaglia depositato presso 1'Archivio di Stato di Torino ${ }^{1}$ si conserva un fascicolo dattiloscritto dal titolo: Auree norme ${ }^{2}$. Il fascicolo si può considerare per certi aspetti come la specificazione tecnica dei Criteri di lavoro già elaborati da Salvatore Battaglia nel 1951 e pubblicati da Bruni (1992), dove si anticipa la stesura delle norme stesse: "Inoltre l'autore verrà precisando con successive norme e schemi i dettagli necessari per la realizzazione pratica e quotidiana del lavoro" (ivi, p. 122) ${ }^{3}$

Le Auree norme risolvono incertezze tipografiche ${ }^{4}$, definiscono come rendere uniformi le sigle o le citazioni, come trattare tecnicismi, esempi o proverbi, ecc., ma non solo: data la struttura del GDLI, le regole riguardano sostanzialmente la definizione della voce e la citazione dei testi. Ogni lemma, infatti, dopo la definizione presenta le citazioni d'autore e termina con l'etimologia.

1 ASTo, Sezioni Riunite, Casa editrice UTET, Archivio della redazione del "Grande Dizionario della lingua italiana" di Salvatore Battaglia (da qui in avanti abbreviato in ASTo, Archivio GDLI).

2 ASTo, Archivio GDLI, Serie 3: Norme redazionali, busta unica, fasc. Auree norme. Ediz. 1984 (da qui in poi abbreviato in ASTo, Auree norme). Nel fascicolo sono presenti due copie dattiloscritte delle norme (ciascuna di una ventina di pagine) con note manoscritte e riproduzioni parziali delle stesse.

3 Per altro i critici, quali Folena (1961), auspicavano una maggiore sistematicità: "Sarebbero particolarmente desiderabili una unificazione progressiva dei criteri di citazione e un indirizzo più sicuro nell'ordinamento semantico" (p. 57).

4 Per la pregevolezza dell'aspetto tipografico dell'opera cfr. Folena (1961, p. 53). 
Nell'archivio il processo di formazione della voce si può seguire punto per punto grazie alle scatole con le schede preparatorie. Si tratta di foglietti manoscritti o dattiloscritti, tutti di uguale formato, con in alto a destra il lemma al quale la scheda si riferisce e al centro la definizione o una citazione d'autore. Queste schede rappresentano la fase che precede la voce di dizionario vera e propria e sono così ordinate: a) una scheda con la definizione; b) schede con citazioni scelte (tendenzialmente in ordine cronologico); c) una scheda etimologica.

Le Auree norme intervenivano proprio su questa fase del lavoro, la revisione e la messa in sequenza delle schede ${ }^{5}$. Sulle schede sono riportati ripensamenti e correzioni in vista della composizione del lemma in bozze in colonna (pronte per la stampa dopo un'ultima lettura).

Il fascicolo delle norme non si sviluppa in una stesura sistematica e organica ${ }^{6}$ ma si presenta come una raccolta, all'apparenza occasiona$\mathrm{le}^{7}$, di indicazioni da applicare durante il lavoro che finiscono per investire gran parte del compito redazionale, compresa la fisionomia della definizione dei lemmi:

La definizione deve evitare il più possibile la semplice serie dei sinonimi. Soprattutto quando si tratta di lemmi concettuali (carità, gioia, libertà, ecc.) la definizione deve effettivamente spiegare tutto il contenuto concettuale, possibilmente con una frase ampia e ben costruita (evitando, cioè, la serie

5 Questo procedimento si manterrà in parte anche quando il lavoro utilizzerà l'elaboratore elettronico: "Il procedimento recentemente adottato per la redazione, mediante elaboratore elettronico, delle voci del Dizionario comprende le seguenti operazioni: A) Redazione: 1) stesura delle voci, a cura di Redattori; 2) revisione e correzione delle stesse, a cura di uno o più Redattori-Correttori; 3) prima revisione generale, con eliminazione degli esempi superflui e/o inserimento dei nuovi a cura del prof. Barberi; B) Segreteria: 4) battitura dattilografica delle definizioni e delle etimologie, a cura della dattilografa; [...]; C) Redazione: 6) revisione definitiva di tutto il materiale battuto, verificato e corretto, a cura del prof. Barberi e del dott. Marchetti" (ASTo, Archivio GDLI, Serie 26: Redazione con elaboratore elettronico, 1979, busta unica).

6 Si veda, ad esempio, la descrizione dei criteri del GRADIT (pubblicati in De Mauro, 2005, pp. 48-112).

7 La prima norma recita: "le parole straniere si registrano quando sono italianizzate nella pronuncia e nella forma" mentre l'ultima "si metta sempre l'etimologia degli avverbi in -mente" (ASTo, Auree norme, pp. 1;20). Si sono riprodotte le sottolineature presenti nel testo in quanto non sempre corrispondenti alla codifica del corsivo. 
delle brevi proposizioni che si accavallano l'una sull'altra). La definizione deve, quando è necessario, sfumare l'eventuale serie delle frasi esplicative in ordine di importanza e di sottigliezza, con l'uso per esempio, della parentesi o di modi come $o$ anche e analoghi. Si eviti quanto più è possibile la rigidità di frasi tutte sullo stesso piano. (p. 4)

Ciò può costituire l'indicazione pratica conseguente a quanto scritto nei Criteri:

Il valore d'un dizionario si misura dalla esattezza e perspicuità delle definizioni e dal discernimento con cui si riesce a distinguere e individuare le diverse accezioni di uno stesso vocabolo. Il primo compito del lessicografo è di «centrare» ogni termine nel suo significato fondamentale e di seguirlo nei suoi vari sensi. [...] Uno dei modi per evitare la definizione generica è di non ricorrere per spiegare un vocabolo ai suoi sinonimi. Cioè si dovrà partire sempre dal convincimento che non esistono veri e propri «sinonimi», ma che ciascuna parola risponde nella coscienza del parlante a una sua precisa e insostituibile funzione. (pp. 122-123)

L'altro aspetto di interesse è la citazione d'autore. Nei Criteri, infatti, si afferma:

Il nostro Dizionario sarà confortato da una larga e continua documentazione di «esempi»: e tutti d'autori. È l'Italia che ha introdotto per la prima nella moderna tradizione lessicografica il costume della «citazione». La fortuna del Tommaseo-Bellini è basata principalmente sulla ricchezza e varietà delle citazioni. (p. 125)

È molto sentita l'esigenza del superamento della dicotomia tra fedeltà al testo e concezione moderna del dizionario. Quello che è considerato un necessario adattamento del testo antico ad una lettura moderna costituisce un capitolo delle norme: 1'Adeguamento testi (pp. 15-20) ${ }^{8}$. Per le edizioni critiche, tuttavia, non si prevedono modifiche:

8 "Un dizionario è subito morto se le parole non sono concretate e rese vive nei diversi usi che ne sono stati fatti: è il problema delle citazioni, che possono essere semplici documenti dell'uso, ma devono essere anche la dimostrazione della ricchezza intellettuale, emotiva, inventiva della letteratura, che è l'impiego più alto, esemplare, durevole della lingua" (Bàrberi Squarotti, 2002, p. 306). 
- Le edizioni critiche non sono soggette ad adeguamenti. Ci si limita a eliminare le $\underline{\mathrm{h}}$ etimologiche (p. 15) ${ }^{9}$.

La scelta di un adeguamento agli usi grafici moderni impone interventi di tipo ortografico, e non solo:

- Le citazioni di edizioni antiche o vecchie vanno, quando è necessario, ammodernate secondo precise regole:

- adattamento della punteggiatura alle consuetudini moderne (cioè: abolizione della virgola prima delle congiunzioni; sostituzione, quando è il caso, del punto e virgola con la virgola o il punto; inserzione del punto interrogativo quando la frase lo richiede, ecc.) (p. 15)

\section{Per quanto riguarda i latinismi grafici, si osserva:}

- la congiunzione et diventa e sempre. Resta et davanti a vocale; ${ }^{10}$

- il nesso -ct di tucto rimane tale e costituisce var. da segnalare dopo il lemma;

- il nesso -pt di descriptione rimane tale (pp. 14-15)

Nulla viene fatto per semplice automatismo, ma è frutto di riflessione semantica o etimologica: "Sono invece considerate varianti le forme ant. in -tade e -tate (bontade, paupertate, ecc.)" (p. 2).

9 Sulla soppressione dell''h' si ritorna: “eliminazione dell'h etimologica (huomo, hora, ecc.);" (ASTo, Auree norme, p. 15). Certo la norma non è nuova se era già presente nella prima edizione del Vocabolario della Crusca (cfr., fra gli altri, Schweickardt 2016, p.512) e trova applicazione nella correzione delle citazioni: "fioriro al mondo, ond'hor sapran le genti [...] sacri thesauri et odorati incensi [...]" è corretto in "fioriro al mondo, ond'or sapran le genti [...] sacri tesauri et odorati incensi [...]" (ASTo, Archivio GDLI, Serie 4: Voci redatte, scatola 1926, lemma 'prèndere', scheda 18525). $\mathrm{Si}$ va anche oltre la ' $h$ ' etimologica: è corretto 'ghauderete' in 'gauderete' e 'ghaudio' in 'gaudio' (ASTo, Archivio GDLI, Serie 4: Voci redatte, scatola 1744, lemma 'per', scheda 133275).

10 Proponiamo il confronto con Serianni (1989): " $E$ può assumere una $d$ eufonica davanti a vocale ("ed avere"); tuttavia, l'uso contemporaneo, almeno quello orale, tende a riservare questa variante, così come avviene per $\mathrm{a} / \mathrm{ad}$, ai casi di incontro con la stessa vocale" (XIV, §13). 
L'esigenza di ammodernamento porta a variazioni che suscitano interrogativi che non si limitano alla sola ortografia.

- le preposizioni articolate scritte staccate si uniscono (a gli diventa agli; de

$\underline{i}$ diventa dei. Ma de le rimane tale: non si raddoppia la $\underline{1}$ );

- ale, dele si scioglie in a le, de le

- come anche su $\underline{i} e \underline{\text { su }}$ gli rimangono tal quali

- $\underline{\text { col resta tale e quale }}{ }^{11}$

- gli antichi apostrofavano gli anche davanti a $\underline{u}, \underline{o}$, a $\underline{\text { e }}$. È necessario, in questi casi, riportare gli alla forma piena; (pp. 16-17) $)^{12}$

Esaminando più nel dettaglio il fascicolo di norme, si trovano indicazioni grammaticali più esplicite. Il GDLI, come ogni dizionario, si è confrontato con questioni morfologiche (oltre all'ovvia necessità di inserire a lemma parti del discorso che hanno soltanto una funzione grammaticale come gli articoli, le preposizioni, ecc.). Questo aspetto accompagna negli anni il lavoro. Nel carteggio tra Salvatore Battaglia e la redazione emerge, ad esempio, la ricorrente menzione della difficoltà nell'affrontare il lemma 'che': "Mi congratulo con lei e gli amici per i vari che ${ }^{13}$ : svolti assai bene"14. Sul 'che' si ritornerà in un'altra lettera:

11 Aggiunto a matita in una sola copia del fascicolo, ma rispettato nelle schede: "sebbene avesse preso con l'aiutante le sue lettere" è corretto in "sebbene avesse preso coll'aiutante le sue lettere" (ASTo, Archivio GDLI, Serie 4: Voci redatte, scatola 1926, lemma 'prèndere', scheda 18859). Restano uguali anche 'pella' = 'per la', 'pello' = 'per lo', 'pel' ='per il', 'pelle' = 'per le' es.: "andare alla virtù pella destra via" (ASTo, Archivio GDLI, Serie 4: Voci redatte, scatola 1744, lemma 'per', scheda 133321), "della Banca pello splendido matrimonio" (ASTo, Archivio GDLI, Serie 4: Voci redatte, scatola 1744, lemma 'per', schede 133341, 133355), "Pel mal ch'avete fatto a torto a quelli; quella notte, pel frastuono, pel giuoco del futuro" (ASTo, Archivio GDLI, Serie 4: Voci redatte, scatola 1744, lemma 'per', schede 133342, 133354, 133406).

12 Le numerose correzioni presenti sulle schede possono certo lasciare perplessi i linguisti e gli storici della lingua che tradizionalmente si sono affidati alla lezione del GDLI.

13 La doppia sottolineatura è nel testo.

14 Lettera di Salvatore Battaglia a Bàrberi Squarotti del 20 ottobre 1962 (ASTo, Archivio GDLI, Serie 16: Corrispondenza fra la Redazione e Salvatore Battaglia, f. 1). Anche la redazione commenta la questione: "il lavoro è continuato regolarmente anche durante l'estate, nonostante le ferie, e abbiamo iniziato in questi giorni la redazione 
Forse avrei voluto iniziare ancora su queste bozze - specie sui vari che. Come le scrissi prima ritengo che le varie voci su questi pronomi e congiunzioni abbiano trovato una felice redazione. Ma qualche perplessità mi è ancora rimasta. In genere, mi lasciano incerto alcune citazioni. Trattandosi di voci così comuni, a me pare che le citazioni avrebbero dovuto avere, tutte, un sapore particolare, almeno nei lemmi d'uso ordinario ${ }^{15}$.

Per maggiore chiarezza espositiva si sono raggruppate le osservazioni secondo le parti del discorso senza tenere conto dell'ordine in cui compaiono nel testo delle Auree norme; i verbi ne raccolgono il maggior numero, a cominciare dalla separazione tra transitivi e intransitivi.

In linea di massima, si distinguano con apposite definizioni i tr. dagli intr. (a meno che non si tratti di casi in cui la forma intransitiva - o transitiva - sia semplicemente un'appendice del significato principale. (p. 6)

Si specifica quando bisognerebbe presentare il paradigma dei verbi:

Si segnalano le prime persone dei verbi la cui sillaba tonica è e o o oppure quando sono sdrucciole (mollifico); quando possono sorgere ambiguità si indica anche la seconda persona per esteso (fortìico, fortìfichi); si dà la coniugazione dei verbi irregolari ma non si segnalano le varianti "storiche" della coniugazione (presono invece di presero, andòe invece di andò, ecc.) (p. 2) ${ }^{16}$.

\section{In effetti il lemma del GDLI riporta:}

Prèndere (ant. pènre, prehèndere, prèndare, prèndre, prènere, prènnere, prìndere), tr. (pass. rem. prési, prendésti, prése; fut. prenderò; part. pass, préso, ant. priso, prenduto). ${ }^{17}$

del CH (con i lemmi difficilissimi CHE, CHI, ecc.)" (Lettera 4 settembre 1961 della redazione a Salvatore Battaglia) (ASTo, Archivio GDLI, Serie 16: Corrispondenza fra la Redazione e Salvatore Battaglia, f. 1).

15 Lettera di Salvatore Battaglia a Bàrberi Squarotti del 5 novembre 1962 (ASTo, Archivio GDLI, Serie 16: Corrispondenza fra la Redazione e Salvatore Battaglia, f. 1).

16 Si sono corretti i refusi evidenti ('delgi' al posto di 'degli', ecc.).

17 GDLI, s.v. préndere. Il paradigma del verbo è già sulle schede rispetto alle quali è stato eliminato prexo dalle bozze a stampa (cfr. ASTo, Archivio GDLI, Serie 9: Bozze in colonna e impaginati, busta 167, f. 167/5, bozza 0871). 
Altri verbi hanno paradigmi più ricchi: ad esempio, 'ruggire' presenta la prima e la seconda forma dell'indicativo presente ${ }^{18}$; 'sapere' oltre alla prima persona dell'indicativo presente menziona la prima del futuro semplice, del passato remoto, del congiuntivo presente, del condizionale presente, del gerundio e del participio passato; per il verbo 'salire' si indicano tutte le persone dell'indicativo presente, la prima persona dell'imperfetto indicativo, del congiuntivo, del condizionale e del participio presente, nonché la prima e la seconda singolare, e infine la terza persona singolare e plurale del passato remoto ${ }^{19}$.

Si propone un confronto con alcune voci del GRADIT, dato il profondo e consapevole legame esistente tra il dizionario moderno e il GDLI; infatti nel GRADIT "è entrato ogni vocabolo presente nel Battaglia (anche se assente in altre fonti), a condizione che non fosse obsoleto e presente come tale solo nel Battaglia" 20 .

Il lemma 'prendere' nel GRADIT fornisce soltanto l'indicazione grammaticale: "v. tr. e intr. (io prendo /'prendo/)".

In alcuni casi la decisione o l'incertezza si verifica sulle schede in cui da una correzione-o successiva aggiunta a penna-si sviluppa il paradigma del verbo o la parte che si desidera esplicitare. Ad esempio, la scheda di 'quadruplicare' inizialmente non prevedeva parti della coniugazione che sono state aggiunte (con accento) per le prime due persone dell'indicativo presente ${ }^{21}$; e quella del verbo 'salire' proponeva anche la

18 E anche la forma antica della terza persona 'rugge', 'ruggono' (GDLI, s.v. ruggire). Per la scelta dei verbi da commentare e confrontare (essendo impensabile oltre che inutile un confronto statistico globale) si sono scelti alcuni verbi o perché citati nelle norme o perché rappresentativi di usi pronominali, servili, con presente indicativo in -isco, ecc.

19 E anche alcune forme antiche o popolari come 'saglia' o 'salisca' (GDLI, s.v. salire).

20 De Mauro, 2005, p. 43. In verità, non si propone in questa sede un confronto sistematico tra i due dizionari, che non avrebbe pertinenza, ma si intende sfruttare il paragone qualora possa offrire spunti interpretativi.

21 Quindi 'quadrùplico', 'quadrùplichi' (cfr. GDLI, s.v. quadruplicare e ASTo, Archivio GDLI, Serie 4: Voci redatte, scatola 2051, scheda 3499). 
prima persona dell'imperfetto indicativo e congiuntivo, ma sono state cassate da un'impietosa penna rossa ${ }^{22}$.

È chiaro, quindi, che oltre ad adempiere allo scopo pratico di fornire il paradigma di verbi irregolari il corredo grammaticale si arricchisce in relazione a casi che hanno stimolato la redazione: ad esempio per il verbo 'sapere' alcune precisazioni grammaticali sono state aggiunte sulle schede in fase di revisione come "anche in relazione con una prop. subord. [...]"23, mentre altre sono state espunte: il punto 3 proponeva "Anche unito a un avv. di quantità o di modo, per indicare l'ampiezza delle nozioni possedute, $[\ldots]$ "24 ed è stato semplificato in "Anche unito con l'indicazione dell'ampiezza delle nozioni possedute, [...]"25.

Altri aspetti non sono menzionati nelle norme e si possono solo desumere. Ad esempio, si nota la tendenza del GDLI a non porre a lemma le forme riflessive o pronominali del verbo, a differenza, invece, del GRADIT, che pone sempre a lemma queste forme deliberatamente ${ }^{26}$. Paradigmatico il caso del verbo 'sapere', che nel GRADIT è strutturato in due occorrenze che separano il verbo dal sostantivo cui si aggiunge un lemma, 'sapersi': "v. pron. intr. [...] [der. di ${ }^{1}$ sapere] con valore copulativo, essere consci di una propria qualità, dote, capacità e sim: [...]" a questa parte si aggiunge anche una specificazione relativa alla coniugazione. Per il verbo 'vedere', il GRADIT pone a lemma anche le

22 Sono stati cassati anche ulteriori esempi di forme antiche e dialettali, es. 'sapi', 'sape', 'sàcciono', 'saprìa', ecc. oltre alla proposta dell'uso sostantivato (cfr. ASTo, Archivio GDLI, Serie 4: Voci redatte, scatola 2421, lemma 'sapére'", schede 68402, 68403).

23 ASTo, Archivio GDLI, Serie 4: Voci redatte, scatola 2421, lemma 'sapére", scheda 68439.

24 ASTo, Archivio GDLI, Serie 4: Voci redatte, scatola 2421, lemma 'sapére', scheda 68540.

25 GDLI, s.v. sapére ${ }^{l}$.

26 "Sono stati considerati verbi pronominali circa cinquemila verbi intransitivi propriamente pronominali, $i$ transitivi che possono essere costruiti con un pronome riflessivo e i verbi pronominali sia transitivi sia intransitivi”" (De Mauro, 2005, p. 94). 
forme 'vedersi' come pronominale intransitivo e con valore copulativo, e 'vedersela' come "v. procompl.".27.

Notiamo che il GRADIT lemmatizza le forme 'prenderle', 'prendersela' e 'prendersi', che nel GDLI sono interne; altrettanto, 'quadruplicarsi' è lemma per il GRADIT ("v. pronom. Intrans") 28 , mentre è interno a 'quadruplicare' per il GDLI "3. Intr. anche con la particella pronom." 29 .

Se non vi sono norme relative al trattamento dei riflessivi o dei pronominali, sono invece presenti delle indicazioni sulle espressioni polirematiche ${ }^{30}$ chiamate dal GDLI semplicemente locuzioni:

Nelle locuzioni si pongano le frasi che, effettivamente, spostano il significato del lemma. Si evitino quelle che sono semplicemente ripetitive della definizione.

$[\ldots]$

Nei verbi le locuzioni devono essere ridotte al minimo e portate sotto i sostantivi relativi. (Sotto i verbi si cerchi di risolvere con definizioni appropriate le forme fraseologiche adottando la formula anche o per lo più nelle espressioni ...) (p. 7).

27 GRADIT, ss.vv. "Figurano a lemma i procomplementari dotati di un significato proprio non riconducibile al verbo principale o molto cristallizzato" (De Mauro, 2005, p. 94).

28 GRADIT, s.v. quadruplicarsi.

29 GDLI, s.v. quadruplicare.

30 "Quelle in cui il valore delle singole parole si trasvaluta e il nesso nella sua globalità appare con un imprevedibile valore nuovo rispetto ai valori dei singoli componenti" (De Mauro, 2005, p. 31). Ricordiamo inoltre Salvi (in Renzi-Salvi, 1991-1995, I, pp. 91-93) e il concetto di 'verbi supporto' tra i quali rientra anche 'prendere' e dove locuzioni fisse rendono il verbo semanticamente neutro mentre la parte semanticamente significativa resta sul complemento; sono citati 'prendere sonno' e 'prendere in odio' (cfr. Salvi, in Renzi-Salvi 1991-1995, I, p. 93). Renzi considera espressioni idiomatiche con valore di solo verbo alcune delle locuzioni formate dal verbo 'prendere' quali 'prendere nota', 'prender sonno', 'prender terra', 'prender fuoco', 'prender servizio' e 'prender tempo' (cfr. Renzi, in Renzi-Salvi, 1991-1995, I, p. 429). Per l'espressione idiomatica 'prendere il toro per le corna' e le limitate possibilità di sostituzione dei sintagmi interni cfr. Salvi (in Renzi-Salvi, 1991-1995, I, p. 90). Per entrambi i dizionari sintagmi come 'prendersi cura' sono classificati come "locuzioni" (e il GRADIT aggiunge "verbale"). 
Alcune forme come 'prendere in castagna' sono inserite già nella definizione del lemma su base semantica ed elencate tra parentesi:

trattare una persona in un certo modo, farla oggetto di un determinato comportamento, in partic. di maltrattamenti (per lo più [aggiunto] nelle espressioni prendere a pugni, a scappellotti, a schiaffi; prendere a ritroso, con le buone, con le molle [aggiunto], prenderlo [corretto in prendere] per il suo verso; sapere prendere uno [forma espunta]; prendere gioco, prendere in giro $)^{31}$.

Più in generale, nella correzione delle definizioni traspare una tendenza alla semplicità: piuttosto che "provocare un'irritazione" è preferito "irritare"32. "usufruire della facoltà concessa dalla Chiesa di ottenere la remissione totale o parziale delle pene temporali compiendo determinate pratiche" è sintetizzato in "lucrare le indulgenze"33.

Naturalmente anche i sostantivi e gli aggettivi cadono sotto le norme:

Il cambio di genere, i plurali regolari o irregolari vanno posti dopo l'indicazione grammaticale e prima dell'indicazione storica o tecnica: $\underline{\text { Pugno }}$, sm. (plur. anche f. le pugna). Letter.; Barbàrico, agg. (plur. m. -cii); Basilisco,

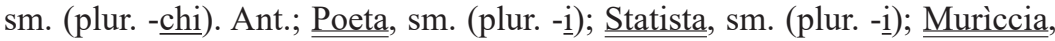
sf. (plur. - $\underline{\text { ce, }}$ raro -cie). (p. 2)

A lemma troviamo applicate le indicazioni; per 'pugno' si legge, infatti:

31 ASTo, Archivio GDLI, Serie 4: Voci redatte, scatola 1926, lemma 'prèndere', scheda 19079.

32 ASTo, Archivio GDLI, Serie 4: Voci redatte, scatole 1926-1927, scheda 18572.

33 ASTo, Archivio GDLI, Serie 4: Voci redatte, scatola 1926, lemma "prèndere', scheda 18709. La tendenza alla semplicità governa comunque: "con uso assoluto" è semplificato in "assol." (cfr. idem, scheda 18520), "lastricati" in "pavimentazioni" (ASTo, Archivio GDLI, Serie 4: Voci redatte, scatola 2051, lemma 'quadrotto', scheda 003363), ecc. 
Pugno (pùgnio), sm. (plur. ant. anche femm. le pugne; ant. e letter. le pugna). Mano le cui dita vengono avvicinate al palmo e strette fortemente, per lo più per afferrare o stringere un oggetto, per impugnare un'arma ecc. ${ }^{34}$

Se si confronta questo lemma con quello del GRADIT il riferimento al plurale femminile non fa parte della definizione e il riferimento a 'le pugna' è rimandato alla nota grammaticale che precede le locuzioni avverbiali (mentre il femminile 'le pugne' è espunto, evidentemente come troppo antico $)^{35}$.

Alcuni plurali incerti per tradizione vengono uniformati:

i plurali di vizio, proprio, ecc. sono vizi, propri, ecc. Si eliminano le doppie ii. Si accenti: prìncipi e princìpi, ecc. e anche dànno quando è voce verbale. Si metta l'accento circonflesso soltanto nei casi in cui neppure l'accento scioglie l'equivoco tra parole diverse (obbligatorî da obbligatorio; obbliga-

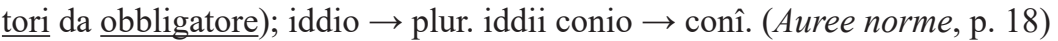

In effetti, nel lemma 'vizio' il plurale non si trova nella definizione, ma nelle citazioni sono presenti le forme plurali: 'vizi'; sei volte la forma 'vizî' ${ }^{36}$ e anche un 'vizii' ${ }^{37}$. Evidentemente le norme non erano sempre tassativamente seguite. Peraltro, non è presente alcun latinismo 'vitio' in osservanza alla norma 'trasformazione del nesso ti + vocale in zi + vocale" (p. 15)

Alcune aggiunte nelle schede riguardano la fonetica: da un iniziale “comp. da 'qua' e 'giù' (v.)" relativo a 'quaggiù' si aggiunge "con raddoppiamento fonosintattico" 39 .

La norma è ancor più sentita nel caso della differenza di apertura della '-e' in verbi e congiunzioni:

34 GDLI, s.v. pugno. Gli esempi sono naturalmente molto numerosi

35 Cfr. GRADIT, s.v. pugno.

36 Risultano correzioni sulle schede: da 'vizii' a 'vizî' (ASTo, Archivio GDLI, Serie 4: Voci redatte, scatola 3238, lemma 'vizio', schede 145420, 145422, 145486, 145487 , 145505, 145515).

37 GDLI, s.v. vizio.

38 Norma già presente nella prima edizione del Vocabolario della Crusca (cfr., fra gli altri, Schweickardt 2016, p. 512).

39 ASTo, Archivio GDLI, Serie 4: Voci redatte, scatola 2051, scheda 3680. 
eliminazione degli accenti su a preposizione e inserzione degli accenti su giacché, perché, né, ecc.; ${ }^{40}$

$[\ldots]$

si faccia molta attenzione agli accenti: é acuti su tutti i composti di che (poiché, perciocché, ecc.), su né, sui composti di re e di tre, ${ }^{41}$

Peraltro, nel lemma 'poiché' si affronta il valore sintattico dell'espressione senza particolare sistematicità, in relazione sinonimica con congiunzioni temporali o causali o con la definizione es.: "3. introduce una prop. concessiva con il verbo al congiunt." o "4. Introduce una prop. interrogativa indiretta" ${ }^{42}$.

Si tratta di una sottolineatura di rango un po' diverso rispetto al semplice refuso ${ }^{43}$, che non viene esplicitato nelle norme ma è già all'attenzione della redazione, raccomandato nelle lettere di Battaglia:

E se qualche nostro amico redattore avrà meno lavoro per la revisione, lei lo preghi di rivedere con più calma le bozze già riviste - perché sono molti i refusi che i lettori comuni non mancano di rilevare con disappunto ${ }^{44}$.

$40 \quad$ ASTo, Auree norme, p. 15.

41 ASTo, Auree norme, p. 17. Vistosi accenti acuti campeggiano su i 'sé' e 'né' delle schede a sostituire gli accenti gravi. Un esempio fra i tanti: "Non ama sé per sé, né il prossimo per sé, né Dio per sé ma ogni cosa ama in Dio" (ASTo, Archivio GDLI, Serie 4: Voci redatte, scatola 1744, scheda 133428). Al lemma 'viceré' (cfr. GDLI, s.v. viceré) sono corretti o aggiunti se mancanti sulle schede, compresa quella etimologica (ASTo, Archivio GDLI, Serie 4: Voci redatte, scatola 3217, schede 129220, 129222 129226, 129228-129231).

42 ASTo, Archivio GDLI, Serie 4: Voci redatte, scatola 1855, schede 89124, 89129. La differenza rispetto ai dizionari contemporanei è vistosa: il GRADIT propone un breve lemma di rilievo quasi soltanto grammaticale, non fosse per la menzione delle marche d'uso cfr. GRADIT, s.v. poiché.

43 Si possono trovare, ad esempio, dei 'qual'è' corretti (ASTo, Archivio GDLI, Serie 4: Voci redatte, scatola 1744, lemma 'per', scheda 133267).

44 Lettera di Salvatore Battaglia del 9 novembre 1961 (ASTo, Archivio GDLI, Serie 16: Corrispondenza fra la Redazione e Salvatore Battaglia, f. 1). Diverse lettere richiamano lo stesso problema: "Adocchiando qua e là le voci, ho sorpreso qualche refuso: un talcone per falcone una mamo per mano. E allora m'è venuto lo sgomento. Forse non ce ne saranno altri. Forse sono inevitabili. Ma dispiace lo stesso". (Lettera di Salvatore Battaglia del 25/1/1961). "Mi raccomando per la revisione. Dobbiamo evitare i refusi - che compaiono nel primo volume purtroppo. Le ho indicato raisio 
Per quanto riguarda gli aggettivi si insiste sul superlativo:

Anche il superlativo degli aggettivi (da fare soltanto se è documentato, cioè non quando è pura forma d'obbligo) va posto (accentato) dopo l'indicazione grammaticale e prima dell'indicazione storica o tecnica; Lène, agg. (superl. Lenìssimo). Letter. (p. 3)

Nei lemmi, in effetti, si menzionano il superlativo o più in generale forme accrescitive anche se lemmatizzate. Per fare un esempio, l'aggettivo 'sapiente' ${ }^{45}$ presenta tanto il superlativo 'sapientissimo', quanto l'accrescitivo 'sapientone' (anche lemmatizzato) e i diminutivi 'sapientino' e 'sapientuccio'. Peraltro, si trovano anche forme avverbiali al superlativo, quale 'sapientissimamente' nel lemma 'sapientemente', nonché il diminutivo 'sapienzina' al lemma 'sapienza'.

Si raccomanda altresì:

I derivati (diminutivi, ecc.) di un lemma non sono necessariamente in ordine alfabetico. Si evitino le accumulazioni caotiche, che mettono tutti i diminutivi insieme. Si segnala fra parentesi il cambiamento di genere rispetto al lemma ordinario: letteróne (sm.). Non si mette l'indicazione di genere se c'è semplice rimando. ${ }^{46}$

Nelle schede traspaiono scelte di spostamento da diminutivo a occorrenza vera e propria: è il caso del diminutivo di 'vizio'>'viziètto' che da un'originaria scheda che lo vedeva accanto a 'viziarèllo' è stato depennato e spostato come occorrenza indipendente mentre è stato aggiunto "vizierèllo" 47 .

[?] al posto di rosario!! (alla voce bocca n. 3) gli errori - se ce ne sono - cadono sotto gli occhi di pochi specialisti - ma i refusi sdegnano il lettore comune..." (Lettera di Salvatore Battaglia del 22/4/1961) (ASTo, Archivio GDLI, Serie 16: Corrispondenza fra la Redazione e Salvatore Battaglia, f. 1).

45 Giusto per puntualizzare, nel GRADIT 'sapiente' è classificato come aggettivo e come sostantivo, mentre il GDLI ne considera degli usi come sostantivati (cfr. GDLI, S.V. sapiènte 2).

46 ASTo, Auree norme, pp. 7-8.

47 ASTo, Archivio GDLI, Serie 4: Voci redatte, scatola 3238, lemma 'vizio', scheda 145592. 
Per quanto riguarda gli aggettivi indefiniti, parte delle osservazioni grammaticali proviene dai suggerimenti apposti alle schede relative alle citazioni d'autore: ad es. nella definizione di qualche. La possibilità che qualche sia seguito da sostantivi plurali si ricava da un paio di schede (una delle quali con una citazione del Bembo) che riportano un appunto a penna "qualche seguito da plurale"48 o solo "(plur.)"49. I redattori non sempre sono sicuri e di fronte ad alcuni casi aggiungono sulle schede delle note con punti interrogativi ${ }^{50}$.

A volte le Auree norme procedono con schemi riassuntivi che illustrano gli errori da evitare:

Si ricordi, in particolare, che:

- l'infinito seguito da compl. ogg. non può essere considerato sostantivato.

- l'aggettivo preceduto da Qualcosa, alcunché, un che di, si considera sempre agg. (e non sost. né sostant.)

- il part. pass. seguito da compl. d'agente è agg. e non verbo (es.: la zia magnetizzata dalla nipote)

- le costruzioni col part. pass. o pres., che riproducono l'abl. assoluto latino sono considerate forme verbali ${ }^{51}$.

- le forme plur. degli agg. non possono essere mai con uso avverb. (p. 6)

48 ASTo, Archivio GDLI, Serie 4: Voci redatte, scatola 2051, lemma 'qualche', schede 03883, 003885.

49 ASTo, Archivio GDLI, Serie 4: Voci redatte, scatola 2051, schede 03886, 003888, 003890, lemma 'qualche'. 'Un caso estremo sono le microstrutture dei lemmi costituiti da preposizioni, pronomi, articoli, esclamazioni: le glosse di questi non possono presentare accezioni simili a quelle delle glosse per nomi o verbi e quindi sono degli elenchi ostensivi dei vari tipi di contesti in cui la preposizione, il pronome, ecc. può essere trovato. La definizione è una parafrasi sinonimica con informazioni metalinguistiche nell'intento di spiegare la funzione di tali parti del discorso" (Marello, 1996, 120).

50 Al lemma 'quello', di fronte ad una citazione che riporta "quello altro" il redattore annota: "Art? l'altro" (ASTo, Archivio GDLI, Serie 4: Voci redatte, scatola 2061, scheda 012308); oppure di fronte a 'quel medesimo' in una citazione di Savonarola: “al? Art.?” (ASTo, Archivio GDLI, Serie 4: Voci redatte, scatola 2061, lemma 'quello', scheda 012309) o al verbo 'sapere' “oppure decisamente Intr?” (ASTo, Archivio GDLI, Serie 4: Voci redatte, scatola 2421, lemma 'sapére"', scheda 68573).

51 "Caratteristici i participi passati assoluti, ossia con un soggetto diverso da quello della reggente (nel caso di un verbo transitivo, si intenda il participio nel va- 
Ad esempio, il participio passato del verbo 'vedere', 'visto', è classificato subito come aggettivo, mentre 'visto', sostantivo, è inserito al punto 4: "Sm. Firma o sigla di approvazione e di presa visione di un'autorità competente" 52 . Il participio passato del verbo 'sapere', 'saputo', è classificato come aggettivo (e se ne presenta anche il superlativo 'saputissimo' e l'accrescitivo 'saputone', non lemmatizzato, e il vezzeggiativo 'saputello' lemmatizzato).

In verità la questione relativa alla natura verbale o aggettivale di infinito e participio sembra sottintendere una dialettica più complessa, eventualmente in relazione agli studi e ai dibattiti sulla nominalizzazione che si svolgevano in quegli anni e che sicuramente erano presenti all'attenzione e alla sensibilità dei redattori ${ }^{53}$, anche se le carte finora esaminate non presentano riferimenti espliciti.

Altri punti delle norme, in effetti, sembrano ripresi da articoli: "le forme verbali tronche di dare e fare sono dè (e non de') e fé (e non fe')" (p.16) potrebbe far eco, ad esempio, a Leone (1969):

lore passivo) [...]. In casi del genere verrebbe fatto di pensare a una continuazione dell'ablativo assoluto latino" (Serianni, 1989, XI, §§ 415-416).

52 GDLI, s.v. vedere ${ }^{1}$.

53 Citiamo, tra le altre, due osservazioni (una di Salvi e una di De Mauro) di tenore simile in occasione di un convegno SLI del 1983 per richiamare il dibattito di allora sull'argomento: “Complemento oggetto preposizionale vs non preposizionale: solo i verbi possono marcare con il caso oggettivo un SN, mentre i nomi possono reggere un SN che abbia rispetto a essi il ruolo di complemento oggetto solo mediante la P di; abbiamo cioè: hanno cacciato Giorgio vs la cacciata di Giorgio. La presenza di un compl. ogg. non preposizionale sarà dunque indice della natura verbale dell'infinito; ma l'affermazione complementare non è vera, almeno per l'italiano moderno, dove non possiamo avere un compl. ogg. introdotto da di dopo un infinito; [...]" (Salvi, 1985 , p. 245). "Il fatto che uno stesso sintagma possa funzionare sia come predicativo che come non predicativo, a seconda dell'intonazione con cui viene enunciato, o che gli si può attribuire leggendolo, mostra che la predicazione non è portata da una sola determinata classe di strutture o, almeno, sequenze morfosintagmatiche. La predicazione è un'operazione che un utente della lingua compie (in produzione) o postula (in riconoscimento) di fronte a frasi anche molto diverse fra di loro dal punto di vista della struttura sintagmatica, frasi con forme verbali di modo finito e frasi senza verbo" (De Mauro-Thornton, 1985, p. 416). 
a me pare di poter suggerire, mentre ancora si oscilla tra tra $\mathrm{ca} / \mathrm{ca}$ 'e $\mathrm{fra} / \mathrm{fra}$, e pie' non è scomparso [...] di estendere l'apostrofo a tutti i monosillabi tronchi (e quindi anche fe' [da fede] e die' [da diede]); (p. 118)

In conclusione, quello che poteva sembrare un semplice manuale di indicazioni tipografiche si rivela ad uno studio più approfondito uno strumento molto più ricco ed articolato. In esso, seppure senza sistematicità, vi si possono trovare giustificazioni a scelte che, dalla semplice correzione delle schede preparatorie, apparirebbero meramente occasionali. Traspare il dibattito della redazione, le incertezze di chi si trovava a lavorare confrontandosi quotidianamente con dubbi, anche di carattere grammaticale, che non sempre negli ambiti più minuti e circostanziati del lessico era in grado di affrontare appieno.

Si rivela, infine, uno strumento interessante di dialogo attraverso il tempo con coloro che hanno lavorato all'opera dando un senso corale ad un monumento lessicografico solo apparentemente monolitico.

\section{BIBLIOGRAFIA}

Bárberi Squarotti, G. (2002). Il vocabolario del Tommaseo come il romanzo della nostra lingua. In G.L. Beccaria \& E. Soletti (eds.), La lessicografia a Torino da Tommaseo al Battaglia (pp. 283-308). Alessandria: Edizioni dell'Orso.

Beltrami, P.G. (2002). Il "Battaglia" visto dal cantiere del "Tesoro della Lingua Italiana delle origini". In G.L. Beccaria, E. Soletti (Eds.), La lessicografia a Torino da Tommaseo al Battaglia (pp. 309-321). Alessandria: Edizioni dell'Orso.

Bruni, F. (1992). La preparazione del Grande dizionario della lingua italiana nel carteggio tra Salvatore Battaglia e Carlo Verde. Medioevo Romanzo, XVIII, 99-133.

Corino Rovano, S. (2015). Dietro le quinte di un grande dizionario: il GDLI e il suo archivio. In R. Nikodinovska (ed.), Parallelismi linguistici, letterari e culturali. Atti del Convegno internazionale, Ohrid 13-14 settembre 2014 (pp. 119-125). Skopje: Edizione dell’Università "Ss. Cirillo e Metodio" di Skopje. 
De Mauro, T. \& Thornton, A.M. (1985). La predicazione: teoria e applicazione all'italiano. In A. Franchi de Bellis \& L.M. Savoia (eds.), Sintassi e morfologia della lingua italiana d'uso. Teorie e applicazioni descrittive (pp. 407-19). Roma: Bulzoni.

De Mauro, T. (2005). La fabbrica delle parole. Il lessico e problemi di lessicologia. Torino: UTET.

Folena, G. (1961). Lingua Nostra, XXII (2), 52-7.

GDLI = Battaglia, S. (1961-2002). Grande Dizionario della Lingua Italiana, 21 voll. Torino: UTET.

GRADIT = De Mauro, T. (1999-2000). Grande dizionario italiano dell'uso. Torino: UTET

Leone, A. (1969). Norme ortografiche: perché po' ma pič? Lingua Nostra, XXX, 117-8.

Marello, C. (1996). Le parole dell'italiano. Lessico e dizionari. Bologna: Zanichelli.

Salvi, G. (1985). L'infinito con l'articolo. In A. Franchi de Bellis \& L.M. Savoia (eds.), Sintassi e morfologia della lingua italiana d'uso. Teorie e applicazioni descrittive (pp. 243-68). Roma: Bulzoni.

Salvi, G. (1991 - 1995). La frase semplice. In L. Renzi \& G. Salvi G. (eds.), Grande grammatica italiana di consultazione (vol. 1, pp. 37-128). Bologna: Il Mulino

Schweickard, W. (2016). La lessicografia. In S. Lubello (ed.), Manuale di linguistica italiana (pp. 509-35). Berlino/Boston: Walter de Gruyter $\mathrm{GmbH}$.

Serianni, L. (1989). Grammatica italiana. Italiano comune e lingua letteraria. Torino: UTET.

Tommaseo, N. \& Bellini, B. (1977). Dizionario della lingua italiana (20 voll). Milano: Rizzoli, (ristampa anastatica dell'ed. 1865).

Terzolo, L. (1986). Il punto sul «Grande dizionario della lingua italiana». In G. Savoca (ed.), Lessicografia, filologia e critica (pp. 45-53). Firenze: Olschki.

Riassunto: Tutte le redazioni di dizionari prevedono delle regole di edizione. Ogni collaboratore, infatti, deve attenersi allo stile previsto dalla pubblicazione. Così chiunque intenda partecipare al lavoro redazionale, viene di solito provvista di un fascicolo più o meno consistente che contiene le norme per scrivere i lemmi.

Naturalmente anche i redattori del GDLI vennero forniti di questo tipo di materiale. Ogni collaboratore riceveva un sottile libretto dal titolo Auree norme cui doveva attenersi. Tuttavia, ad un esame attento 
di questo fascicolo, le indicazioni previste dal GDLI appaiono più ricche ed articolate. Infatti, non contengono soltanto un insieme di regole formali e di indicazioni tipografiche, ma hanno un respiro molto più ampio. Il redattore si trova di fronte ad una serie di regole relative all'ortografia e alla grammatica. La presente ricerca intende individuare le norme di interesse grammaticale e alcuni elementi di morfologia e sintassi emersi durante la discussione della redazione.

Parole chiave: grammatica, dizionario, lessico, regole, italiano 